\title{
Carbon monoxide stimulates global protein methylation via its inhibitory action on cystathionine $\beta$-synthase
}

\author{
Takehiro Yamamoto, Naoharu Takano, Kyoko Ishiwata and Makoto Suematsu* \\ Department of Biochemistry, School of Medicine, Keio University, JST, ERATO Suematsu Gas Biology Project, 35 Shinanomachi, Shinjuku-ku, Tokyo 160-8582, Japan
}

(Received 8 November, 2010; Accepted 10 November, 2010)

\begin{abstract}
Although carbon monoxide derived from heme oxygenase has been reported to exert diverse biological actions in mammals, macromolecules responsible for its direct reception and functional outcomes of the gas binding remain largely unknown. Based on our previous results in vivo suggesting carbon monoxide serves as an inhibitor of cystathionine $\beta$-synthase that rate-limits transsulfuration pathway for generation of hydrogen sulfide, we have herein hypothesized that the gas might serve as a regulator of protein methylation through accelerating turnover of remethylation cycle residing at the upstream of the enzyme. Metabolomic analysis in human monoblastic leukemia U937 cells in culture revealed that application of carbon monoxide-releasing molecules caused increases in methionine and S-adenosylmethionine and a decrease in cystathionine in the cells, suggesting the cystathionine $\beta$ synthase inhibition by carbon monoxide. Under these circumstances, the cells exhibited global protein arginine methylation: this event was also reproduced by the cell treatment with hemin, a heme oxygenase-1 inducer. The protein arginine methylation elicited by carbon monoxide was attenuated by knocking down cystathionine $\beta$-synthase with its small interfering RNA or by blocking S-adenosylhomocysteine hydrolase with adenosine dialdehyde, suggesting remethylation cycling is necessary to trigger the methylation processing. Furthermore, proteins undergoing the carbon monoxide-induced arginine methylation involved histone $\mathrm{H} 3$ proteins, suggesting chromatin modification by the gas. Collectively with our studies in vivo showing its inhibitory action on endogenous hydrogen sulfide production, the current results suggest that not only inhibition of transsulfuration pathway for $\mathrm{H}_{2} \mathrm{~S}$ generation but also activation of protein methylation accounts for notable biological actions of carbon monoxide via the cystathionine $\beta$-synthase inhibition.
\end{abstract}

Key Words: carbon monoxide, cystathionine $\beta$-synthase, hydrogen sulfide, methylation, epigenetic regulation

C arbon monoxide (CO) generated from heme oxygenase (HO) has the ability to exert diverse biological actions including vascular relaxation and neural transmission. ${ }^{(1-3)}$ The ability of the gas to modestly activate heme-containing soluble guanylate cyclase appears to contribute to such biological actions. $\mathrm{CO}$ also possesses the activity to regulate intracellular metabolism, cell cycle, apoptosis, and proliferation/hypertrophy; these actions are attributable to its ability to modulate mitogen-activated protein kinase (MAPK) system, ${ }^{(4)}$ although the direct macromolecular targets responsible for its functional regulation remain largely unknown. Many investigators have considered a possibility that pleiotropic effects of $\mathrm{CO}$ cannot be explained only by the activation of soluble guanylate cyclase and the novel targets for the $\mathrm{CO}$ binding might exist.
Since macromolecules possessing metal-centered prosthetic groups such as enzymes in metabolic systems might serve as targets for covalent binding of molecular oxygen or $\mathrm{CO}$, we have recently attempted to mine gas-responsive enzymes through reading out alterations in metabolites using metabolome analyses based on capillary electrophoresis assisted by mass spectrometry (CE-MS) in varied experimental models where $\mathrm{O}_{2}$ or $\mathrm{CO}$ is largely altered. ${ }^{(5-9)}$ In the model displaying stress-inducible $\mathrm{CO}$ up-regulation, cystathionine $\beta$-synthase (CBS) turned out to serve as a unique heme protein that is inhibited by $\mathrm{CO}$ in vivo. ${ }^{(5,8)}$ Our metabolomic analyses previously revealed that accumulation of metabolites in the remethylation cycle and contrast downregulation of metabolites in the transsulfuration pathway in the acetoaminophen (AAP)-induced hepatotoxicity model. ${ }^{(6)}$ Since $\mathrm{CO}$ is overproduced through heme oxygenase in the liver, ${ }^{(10)}$ the data led us to hypothesize that $\mathrm{CO}$ alters these metabolites by binding one of enzymes in and around methionine-cysteine metabolic pathways and that a similar alteration in metabolic footprints takes place in other experimental models where $\mathrm{CO}$ is overproduced. This hypothesis was proven in the model of hemeoverloaded mouse liver, ${ }^{(8)}$ showing that $\mathrm{CO}$-overproducing liver down-regulates endogenous $\mathrm{H}_{2} \mathrm{~S}$ to trigger the $\mathrm{HCO}_{3}{ }^{-}$-dependent choleresis through the CBS inhibition in vivo.

Although the fact that $\mathrm{CO}$ but not nitric oxide (NO) inhibits CBS has well been demonstrated in vitro, ${ }^{(11,12)}$ its physiologic and pathophysiologic impacts in biological systems remain to be further investigated. In this study, we aimed to examine if stressinducible $\mathrm{CO}$ exerts its diverse biological actions through alterations in remethylation cycle that determine methylation of macromolecules through limiting metabolic flow towards transsulfuration pathway. The current results suggest that $\mathrm{CO}$ stimulates global protein methylation through its inhibitory action on CBS in cell culture.

\section{Materials and Methods}

Cell culture and reagents tested. A human monoblastic leukemia cell line, U937 cells were maintained in RPMI1640 medium (Invitrogen, Carlsbad, CA) containing 10\% heatinactivated fetal bovine (Invitrogen) and $1 \times$ penicillin/streptomycin (Invitrogen) at $37^{\circ} \mathrm{C}$ in an atmosphere of $5 \% \mathrm{CO}_{2} / 95 \%$ air. Tricarbonyldichlororuthenium (II) dimer (CO-releasing molecule, CORM) and ruthenium (III) chloride as a negative control were purchased from Sigma Aldrich (St. Louis, MO) (\#288144 and \#208523, respectively), were dissolved in dimethylsulfoxide and adding to cells in various conditions. Oxidized adenosine

*To whom correspondence should be addressed. E-mail: msuem@sc.itc.keio.ac.jp 


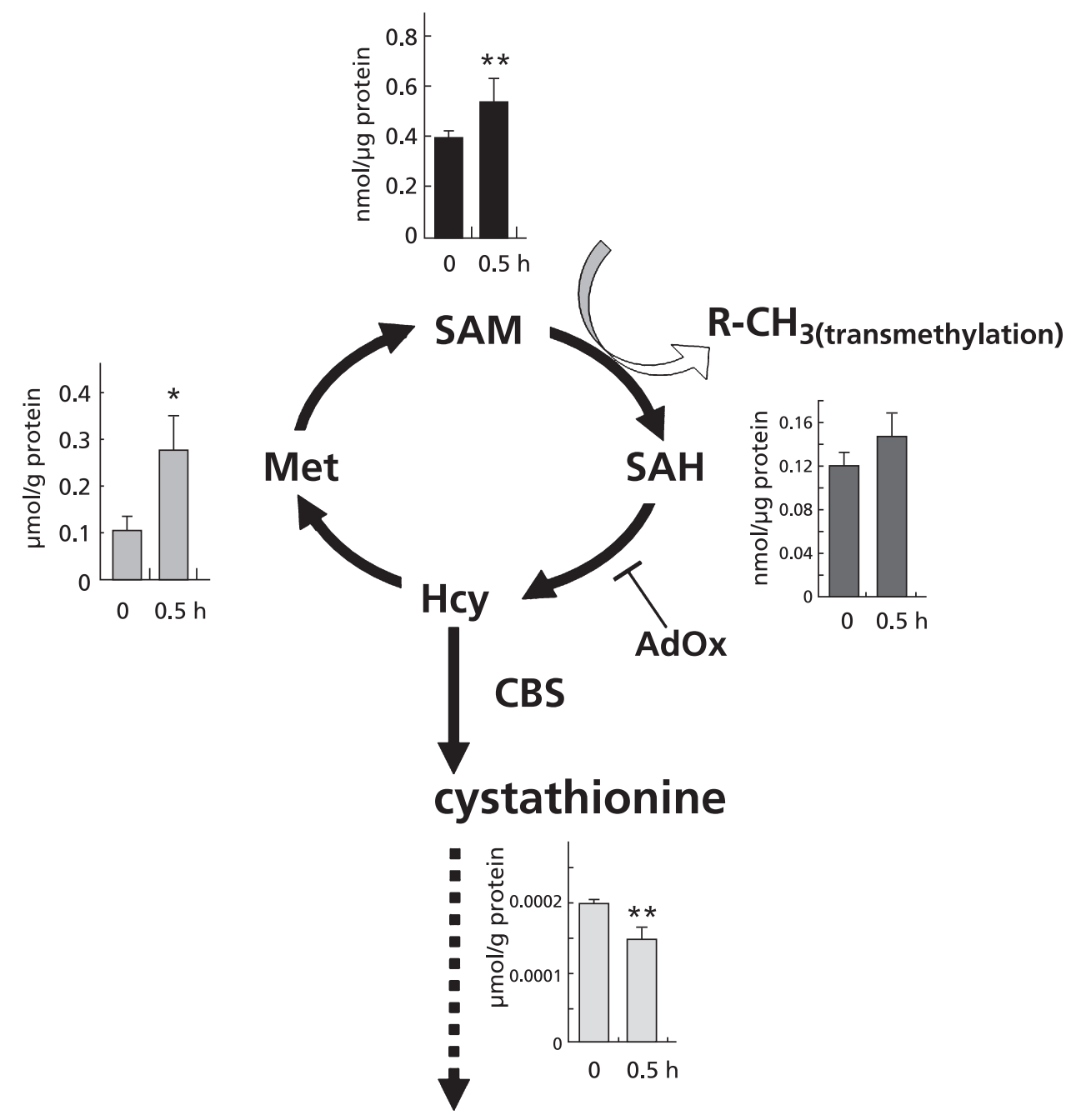

Fig. 1. CO causes the dissociation of the metabolism between remethylation cycle and transsulfuration pathway. Metabolomic analysis concerned with sulfur-containing amino acids was performed with CO-releasing molecule (CORM)-treated U937 cells. Metabolites were extracted at time 0 and $0.5 \mathrm{~h}$ after $100 \mu \mathrm{M}$ CORM administration. Data represent the mean \pm SD of samples from 4 independent experiments. Asterisks indicate statistically significant differences (unpaired Students't test). *: $p<0.05 ; * *: p<0.01$. AdOX; oxidized adenosine, CBS; cystathionine $\beta$-synthase, SAH; S-adenosylmethionine, SAM; S-adenosylcysteine.

(AdOx), a blocker of S-adenosylhomocysteine (SAH) hydrolase that accumulates SAH to inhibit methyltransferases, ${ }^{(13)}$ and hemin (Sigma) were also added to cells at desired concentrations.

Small interfering RNA (siRNA) transfection in U937 cells. The siRNA oligonucleotides for the control (Non-targeting) and CBS (Dharmacon, Inc. Lafayette, CO) were transfected with nucleofactor II instrument (AMAXA biosystem, Germany) into the U937 cells. Briefly, $1 \times 10^{6}$ cells were suspended with $100 \mu \mathrm{l}$ solution $\mathrm{V}$ and mixed with individual siRNAs $(3 \mu \mathrm{g})$. Cells transfected with siRNA, targeting for CBS were harvested for $48 \mathrm{~h}$ and $100 \mu \mathrm{M}$ ruthenium chloride (III) or CORM for $6 \mathrm{~h}$. The cells transfected with siRNA for HO-1, following to incubation for $48 \mathrm{~h}$, were treated with $20 \mathrm{ng} / \mathrm{ml}$ PMA for $6 \mathrm{~h}$.

Measurements of sulfur-containing amino acids. Frozen pellet of U937 cells $\left(1 \times 10^{6}\right)$ were homogenized in $10 \%$ TCA with $10 \mathrm{mM}$ DTPA and following centrifugation briefly and the supernatant was used as a sample. The measurement of intracellular S-adenosylcysteine (SAM) and SAH was according to the previous description using a stable-isotope-dilution liquid chromatography-mass spectrometry (LC-MS). ${ }^{(14,15)}$ The contents of intracellular methionine and cystathionine were determined based on derivatization with AccQ-Fluor ${ }^{\mathrm{TM}}$ kit according to the manufacture's manual (Waters, Milford, MA). The values were normalized by protein concentrations.

Antibody and western blotting. For protein methylation analyses, equal amounts of protein $(25 \mu \mathrm{g})$ were separated by 5 $20 \%$ SDS-PAGE and subsequently transferred to nitrocellulose membranes (GE healthcare UK Ltd., little Chalfont, UK). The membranes were blocked in 3\% skimmed dry milk in PBST for $1 \mathrm{~h}$, incubated with primary antibodies [1:5000 dilution for ASYM24 from Upstate, Charlottesville, VA, \#07-414; 1:5000 dilution for anti-CBS from Abnova (Heidelburg, Germany) \#H00000875-M01; 1:5000 dilution for anti-HO-1 from Stressgen (Victoria, Canada), 1:5000 dilution for anti-GAPDH from Santa Cruz biotechnology, Inc., CA (\#sc-20357)] for overnight at $4^{\circ} \mathrm{C}$. To assess the methylation level of histone $\mathrm{H} 3$, several antibodies, recognize the distinct sites of methylated residue were applied: anti-histone H3 (1:5000 dilution, Santa Cruz, \#sc-10809), antimethyl-histone H3 (1:5000 dilution, \#07-030 for methyl-Lys4; 1:5000 dilution, \#07-352 for methyl-Lys9; 1:1000 dilution, \#07-214 for methyl-Arg17 from Upstate). Following to the incubation for primary antibody, membranes were washed three times in PBST, then incubated with 1:25000 dilution secondary antibody (anti-mouse or rabbit $\mathrm{IgG}$ horse radish peroxidase 
A

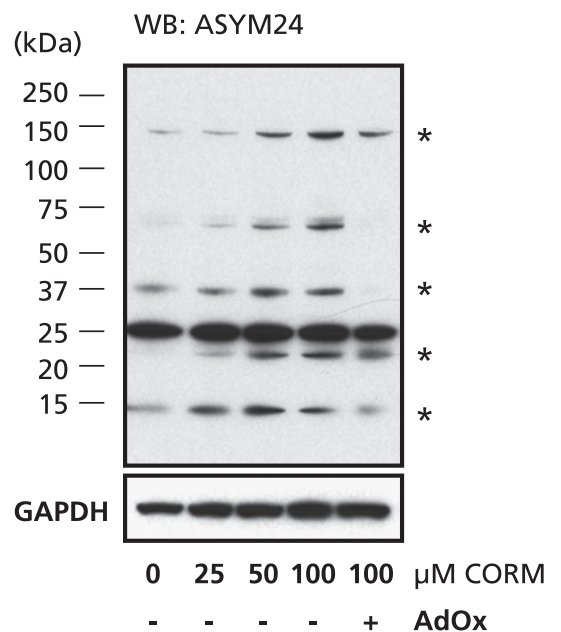

B

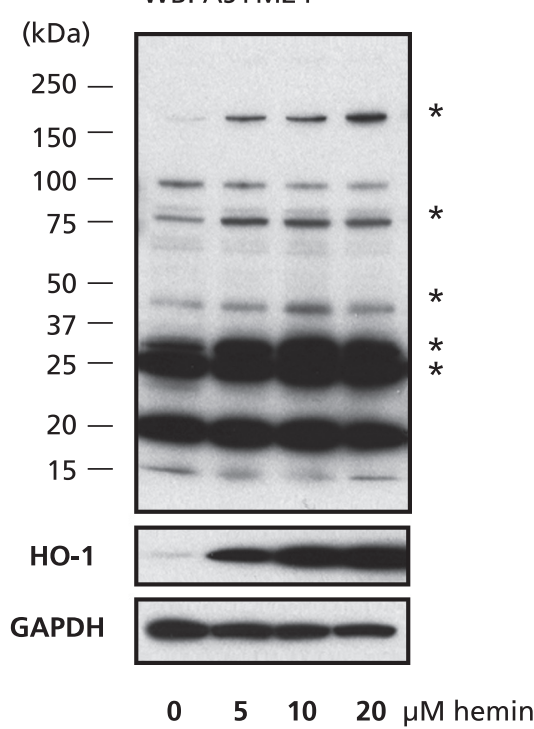

D

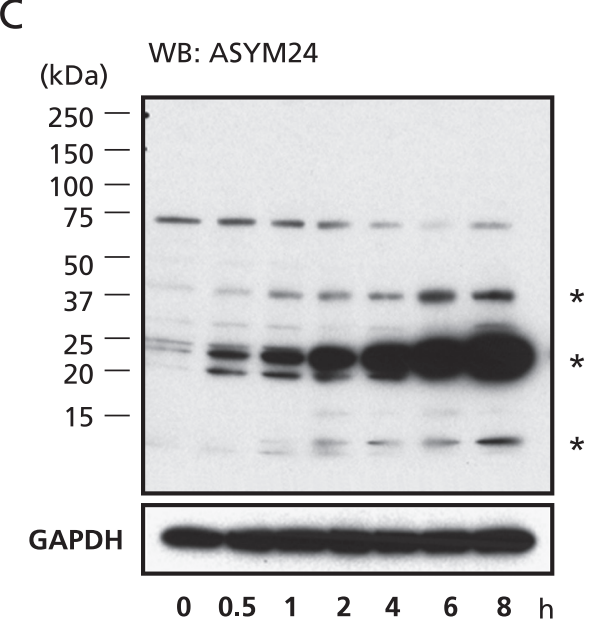

\section{WB: ASYM24}

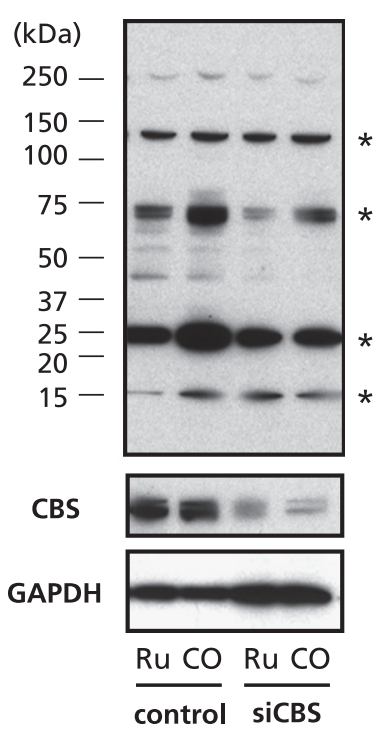

Fig. 2. CO activates protein methylation via CBS activity. A: U937 cell extracts were prepared from grown in the presence of $0,25,50$ or $100 \mu M$ CO-releasing molecule (CORM) 2 for $8 \mathrm{~h}$. Increased methylation level of protein was evaluated with ASYM24, anti-asymmetric dimethylarginine antibody. Asterisks indicate the bands changing the intensity with CORM-treatment. B: U937 cells were treated with $0,5,10,20 \mu \mathrm{M}$ hemin for $8 \mathrm{~h}$. Cell lysate $(25 \mu \mathrm{g})$ was separated by SDS-PAGE and immunoblotted with ASYM24 antibody and anti-heme oxygenase-1 (HO-1) antibody. Asterisks indicate the bands changing the intensity with hemin-treatment. C: U937 cells were exposed with $100 \mu \mathrm{M}$ CORM for 0, $0.5,1,2,4,6$ or $8 \mathrm{~h}$. Extracted protein was separated by SDS-PAGE, and immunoblotted with ASYM24 antibody. Asterisks indicate the bands changing the intensity with CORMtreatment. D: U937 cells were transfected with $3 \mu \mathrm{g}$ of siRNA for non-targeting or CBS for $48 \mathrm{~h}$ as described in Materials and Methods. After further treatment with $100 \mathrm{mM}$ of Ruthenium chloride (III) (Ru) or CORM-2 (CO) for $6 \mathrm{~h}$, levels of total protein methylation were detected using western blotting with ASYM24 antibody. The expression level of GAPDH was determined as an internal control. Asterisks indicate the bands changing the intensity with CORM-treatment. These are representative pictures of 5 separate experiments.

conjugate from GE healthcare) in blocking solution for $1 \mathrm{~h}$. Chemiluminescent detection was performed using the Immobilon Western kit according to the manufacturer's instructions (Millipore, Billerica, MA).

\section{Results and Discussion}

CO increases methionine and S-adenosylmethionine in cells. We examined alterations in metabolites of remethylation and transsulfuration pathways in U937 cells in response to CORM or its control substance $\mathrm{RuCl}_{3}$. As seen in Fig. 1, CORM treatment at $100 \mu \mathrm{M}$ for $30 \mathrm{~min}$ caused a significant decrease in cystathionine, a product of CBS, while causing an increase in methionine and SAM in remethylation cycle. SAH displayed a modest elevation in response to CORM but with no statistical significance. These results suggest that $\mathrm{CO}$ possesses the inhibitory effect on transsulfuration, being in agreement with our previous results showing responses of liver metabolites in 
acetaminophen-induced injury or hemin-overloaded models, ${ }^{(6,8)}$ and such an effect of the gas appeared to influence on cellular levels of the remethylation substrates.

$\mathrm{CO}$ or HO-1 induction causes global protein arginine methylation. To examine if the increase in remethylation substrates by $\mathrm{CO}$ is linked with protein methylation, we performed western blot analyses with ASYM24 antibody, which specifically recognizes asymmetric dimethylated-arginine (ADMA). ${ }^{(16)}$ As shown in Fig. 2A, CORM enhanced the intensity of several different bands detected by ASYM24 antibody in a dose-dependent manner in U937 cells (asterisks indicate the bands changing with CORM treatment). Moreover, AdOx, a SAH hydrolase inhibitor to stop remethylation cycle attenuated the $\mathrm{CO}$-induced protein arginine methylation. Such effects of exogenously applied CO were reproduced by treatment with hemin which dose-dependently induced HO-1 (asterisks in Fig. 2B). These data suggested that $\mathrm{CO}$ increases protein-arginine-methylation via acceleration of remethylation cycle. Fig. $2 \mathrm{C}$ showed the time-dependent increase in ADMA-modified proteins by applying CORM, indicating that enhancement of the arginine methylation appeared to vary among different protein targets. Actually the protein at approximately $75 \mathrm{KDa}$ displayed the maximum signal at 30-60 min after the CORM application that was followed by a gradual downregulation. Although the reasons why CORM decreased ADMA modification in selected proteins in a certain period after the $\mathrm{CO}$ application in the current study, it might depend on differences in the enzymatical regulation of specificity of methyltransferases, cellular localization of metabolic changes or trans-methylation reaction from one protein to another.

To examine whether CBS is involved in the protein methylation modulated by $\mathrm{CO}$, we introduced siRNA targeting for CBS into U937 cells. Compared with non-targeting siRNA transfected cells, CBS knock-down cells became insensitive to CORM for the arginine methylation (Fig. 2D). These data suggested that $\mathrm{CO}$ modulates protein arginine methylation through the inhibition of CBS in the cells.

$\mathrm{CO}$ enhances histone $\mathrm{H} 3$ protein methylation. The current results led us to hypothesize that $\mathrm{CO}$ changes methylation status of nuclear proteins. Therefore, we assessed the nuclear methylation status in the CORM-treated cells by determining the methylation of histone $\mathrm{H} 3$, since this protein is a representative methylated protein at multiple sites including lysine and arginine residues. ${ }^{(17)}$ As seen in Fig. 3, we examined the representative methylation residues of Lys4, Lys9, and Arg 17 on histone H3. Western blot for anti-methylated histone $\mathrm{H} 3$ antibodies revealed that all of three residues were hyper-methylated by CORM. The CO-elicited hypermethylation was inhibited by the $\mathrm{CO}$-incubation with AdOx. These results suggested that $\mathrm{CO}$ enhances methylation levels of histone $\mathrm{H} 3$, raising a possibility that the gas exerts epigenetic modification.

Although the current study has not identified individual protein targets methylated by $\mathrm{CO}$, the results first demonstrated that the gas has the ability to trigger methylation of different protein targets in varied durations. Furthermore, this event appeared to depend on the ability of the gas to inhibit CBS that might trigger altered levels of remethylation substrates in the cells. Further investigation is obviously necessary to identify these protein targets and to examine their functional link to diverse biological

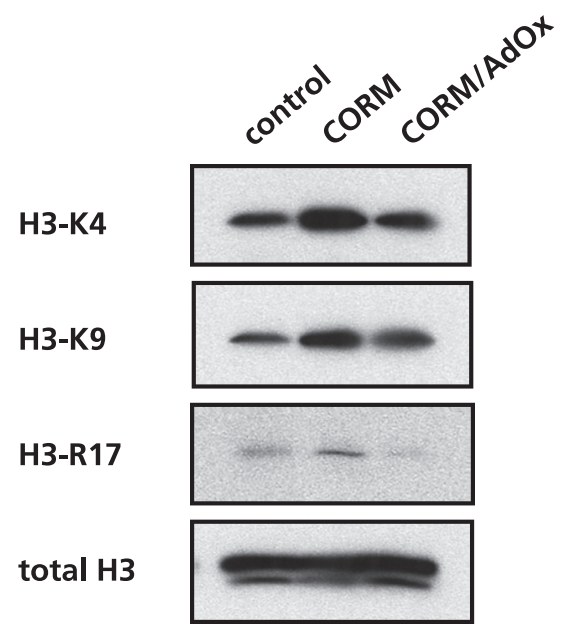

Fig. 3. CO alters the methylation status of histone H3. Cell lysates ( $25 \mu \mathrm{g}$ protein per lane) from U937 cells were treated with CO-releasing molecule (CORM) for $4 \mathrm{~h}$ was separated by SDS-PAGE and performed western blot analyses with several antibodies recognized with distinct methylated residue of histone $\mathrm{H} 3$ described in Materials and Methods. The expression level of histone H3 protein was determined as an internal control. The data show a representative set from 3 independent experiments.

functions that have already been demonstrated in vivo, being underway in our laboratory.

\section{Acknowledgments}

We appreciated the technical supports and advice by Dr. Adachi T. for measurements of metabolites with LC/MS. This work was supported in part by JSPS Grant-in-aid for Creative Scientific Research Grant 17GS0419 (M.S.) and Grant-in-Aid for Scientific Research (18770178) (T.Y.) and Scientific Frontier Research (T.Y.) from the Ministry of Education, Culture, Sports, Science, ant Technology (MEXT). M.S. is the Leader, JST ERATO Suematsu Gas Biology Project.

\section{Abbreviations}

\section{AAP acetoaminophen}

ADMA asymmetric dimethylated-arginine

AdOx oxidized adenosine

CBS cystathionine $\beta$-synthase

CE-MS capillary electrophoresis assisted by mass spectrometry

$\mathrm{CO}$ carbon monoxide

CORM CO-releasing molecule

HO heme oxygenase

LC-MS liquid chromatography assisted by mass spectrometry

MAPK mitogen-activated protein kinase

NO nitric oxide

SAH S-adenosylhomocysteine

SAM S-adenosylcysteine

SiRNA small interfering RNA

\section{References}

1 Verma A, Hirsch DJ, Glatt CE, Ronnett GV, Snyder SH. Carbon monoxide: a putative neural messenger. Science 1993; 259: 381-384.

2 Suematsu M, Kashiwagi S, Sano T, Goda N, Shinoda Y, Ishimura Y. Carbon monoxide as an endogenous modulator of hepatic vascular perfusion. Biochem Biophys Res Commun 1994; 205: 1333-1337.

3 Suematsu M, Goda N, Sano T, and et al. Carbon monoxide: an endogenous modulator of sinusoidal tone in the perfused rat liver. J Clin Invest 1995; 96:

2431-2437.

4 Ryter SW, Alam J, Choi AM. Heme oxygenase-1/carbon monoxide: from basic science to therapeutic applications. Physiol Rev 2006; 86: 583-650.

5 Kajimura M, Fukuda R, Bateman RM, Yamamoto T, Suematsu M. Interactions of multiple gas-transducing systems: hallmarks and uncertainties of $\mathrm{CO}$, $\mathrm{NO}$, and $\mathrm{H}_{2} \mathrm{~S}$ gas biology. Antioxid Redox Signal 2010; 13: 157-192.

6 Soga T, Baran R, Suematsu M, and et al. Differential metabolomics reveals 
ophthalmic acid as an oxidative stress biomarker indicating hepatic glutathione consumption. J Biol Chem 2006; 281: 16768-16776.

7 Kinoshita A, Tsukada K, Soga T, and et al. Roles of hemoglobin allostery in hypoxia-induced metabolic alterations in erythrocytes: simulation and its verification by metabolome analysis. J Biol Chem 2007; 282: 10731-10741.

8 Shintani T, Iwabuchi T, Soga T, and et al. Cystathionine $\beta$-synthase as a carbon monoxide-sensitive regulator of bile excretion. Hepatology 2009; 49: $141-150$.

9 Sakuragawa T, Hishiki T, Ueno Y, and et al. Hypotaurine is an energy-saving hepatoprotective compound against ischemia-reperfusion injury of the rat liver. J Clin Biochem Nutr 2010; 46: 126-134.

10 Mori M, Suematsu M, Kyokane T, and et al. Carbon monoxide-mediated alterations in paracellular permeability and vesicular transport in acetaminophen-treated perfused rat liver. Hepatology 1999; 30: 160-168.

11 Taoka S, Banerjee R. Characterization of NO binding to human cystathionine beta-synthase: possible implications of the effects of $\mathrm{CO}$ and NO binding to the human enzyme. J Inorg Biochem 2001; 87: 245-251.
12 Puranik M, Weeks CL, Lahaye D, and et al. Dynamics of carbon monoxide binding to cystathionine $\beta$-synthase. J Biol Chem 2006; 281: 13433-13438.

13 Johnson BA, Najbauer J, Aswad DW. Accumulation of substrates for protein L-isoaspartyl methyltransferase in adenosine dialdehyde-treated PC12 cells. J Biol Chem 1993; 268: 6174-6181.

14 Stabler SP, Allen RH. Quantification of serum and urinary S-adenosylmethionine and S-adenosyl homocysteine by stable-isotope-dilution liquid chromatography-mass spectrometry. Clin Chem 2004; 50: 365-372.

15 Tian J, Bryk R, Itoh M, Suematsu M, Nathan C. Variant tricarboxylic acid cycle in Mycobacterium tuberculosis: identification of alpha-ketoglutarate decarboxylase. Proc Natl Acad Sci USA 2005; 102: 10670-10675.

16 Boisvert FM, Côté J, Boulanger MC, Richard S. A proteomic analysis of arginine-methylated protein complexes. Mol Cell Proteomics 2003; 2: 13191330.

17 Davie JK, Dent SY. Transcriptional Control: an activating role for arginine methylation. Curr Biol 2002; 12: R59-R61. 